\title{
Modeling the Effects of Age and Sex on Normal Pediatric Brain Metabolism Using 18F-FDG PET/CT
}

\author{
Sophie Turpin ${ }^{1}$, Patrick Martineau ${ }^{2}$, Marc-André Levasseur ${ }^{3}$, and Raymond Lambert ${ }^{1}$ \\ ${ }^{I}$ Division of Nuclear Medicine, Department of Medical Imaging, Centre Hospitalier Universitaire Sainte-Justine, Montréal, Québec, \\ Canada; ${ }^{2}$ Division of Nuclear Medicine, Department of Medicine, University of Ottawa and Ottawa Hospital, Ottawa, Ontario, \\ Canada; and ${ }^{3}$ Department of Nuclear Medicine, Centre Hospitalier Universitaire de Sherbrooke, Sherbrooke, Québec, Canada
}

Reference databases of pediatric brain metabolism are uncommon, because local brain metabolism evolves significantly with age throughout childhood, limiting their clinical applicability. The aim of this study was to develop mathematic models of regional relative brain metabolism using pediatric ${ }^{18} \mathrm{~F}-\mathrm{FDG}$ PET with $\mathrm{CT}$ data of normal pediatric brains, accounting for sex and age. Methods: PET/CT brain acquisitions were obtained from 88 neurologically normal subjects, aged 6 mo to $18 \mathrm{y}$. Subjects were assigned to either a development group $(n=59)$ or a validation group $(n=29)$. For each subject, commercially available software was used to quantify the relative metabolism of 47 separate brain regions using whole-brainnormalized (WBN) and pons-normalized (PN) activity. The effects of age on regional relative brain metabolism were modeled using multiple linear and nonlinear mathematic equations, and the significance of sex was assessed using the Student $t$ test. Optimal models were selected using the Akaike information criterion. Mean predicted values and $95 \%$ prediction intervals were derived for all regions. Model predictions were compared with the validation dataset, and mean predicted error was calculated for all regions using both WBN and PN models. Results: As a function of age, optimal models of regional relative brain metabolism were linear for 9 regions, quadratic for 13 , cubic for 6 , logarithmic for 12 , power law for 7 , and modified power law for 2 using WBN data and were linear for 9 , quadratic for 25 , cubic for 2 , logarithmic for 6 , and power law for 4 using PN data. Sex differences were found to be statistically significant only in the posterior cingulate cortex for the WBN data. Comparing our models with the validation group resulted in $94.3 \%$ of regions falling within the $95 \%$ prediction interval for WBN and $94.1 \%$ for PN. For all brain regions in the validation group, the error in prediction was $3 \% \pm 0.96 \%$ using WBN data and $4.72 \% \pm 1.25 \%$ when compared with the PN data $(P<0.0001)$. Conclusion: Pediatric brain metabolism is a complex function of age and sex. We have developed mathematic models of brain activity that allow for accurate prediction of regional pediatric brain metabolism.

Key Words: FDG; PET; pediatric; brain; normal database; metabolism

J Nucl Med 2018; 59:1118-1124

DOI: 10.2967/jnumed.117.201889

Received Sep. 6, 2017; revision accepted Nov. 30, 2017.

For correspondence contact: Sophie Turpin, Division of Nuclear Medicine, Department of Medical Imaging. Centre Hospitalier Universitaire SainteJustine, 3175 Chemin de la Côte-Sainte-Catherine, Montréal, Québec, Canada H3T 1 C5.

E-mail: turpinsop@hotmail.com

Published online Dec. 28, 2017.

COPYRIGHT @ 2018 by the Society of Nuclear Medicine and Molecular Imaging.
I t is well known that normal pediatric brain development is characterized by significant age- and sex-related variations in local metabolism (1-10) that can render the visual interpretation of clinical brain ${ }^{18} \mathrm{~F}$-FDG PET studies in children challenging, with normal variations in local metabolism potentially confounding pathologic changes.

It is common practice in the interpretation of adult brain PET studies to compare results with reference databases, a technique that has been shown to increase diagnostic accuracy for both focal lesions and neurodegenerative conditions (11-14). This technique has met with moderate success in the case of pediatric studies, for which the availability of reference pediatric databases is limited. This limitation is largely due to two factors. The first is that, because of ethical concerns, dedicated brain PET studies are rarely performed on healthy pediatric patients, severely limiting the ability to develop reference databases. The second is that local brain metabolism evolves drastically throughout childhood, so that no single reference database would be applicable throughout the pediatric age range. This issue can be mitigated by dividing the pediatric age range into several narrow age intervals (e.g., 0-1 y, 2-3 y, and 4-5 y), each with a separate, age-appropriate reference database; however, in light of the first restriction, and the need to have statistically significant numbers in each reference database, such an approach would likely be prohibitive.

In contrast to the use of reference databases, an alternate approach is possible. To date, a few studies have developed ageand sex-dependent mathematic models of local brain metabolism $(4,6,9,10)$. Some of these models have used dedicated brain studies $(3,4,7)$ or the brain component of whole-body PET studies on patients without central nervous system disease (studies that do not show central nervous system involvement or neurologic symptoms) $(6,9)$, whereas others have used dedicated brain PET studies of patients with epilepsy $(1,10)$. Using the results of brain ${ }^{18} \mathrm{~F}-\mathrm{FDG}$ PET in neurologically intact patients of varying age and sex, mathematic models can be developed that predict normal local brain metabolism. Compared with databases, models have the advantage of being able to interpolate results for parameters (i.e., a particular age or sex) that may not be well represented in a limited database. In other words, these models may have the potential to capture variations in metabolism due to age and sex, without the need to scan large numbers of neurologically normal pediatric patients to establish age- and sex-specific databases.

In this paper, we describe the application of commercially available software to develop mathematic models of regional relative brain metabolism using both whole-brain-normalized 
(WBN) and pons-normalized (PN) data. The accuracy of the models was assessed by comparing model predictions with a validation group of neurologically normal pediatric subjects.

\section{MATERIALS AND METHODS}

This retrospective study was approved by our institutional review board, and the requirement to obtain informed consent was waived.

\section{Population}

Seven hundred twenty pediatric patients referred to our institution between April 2008 and June 2017 for dedicated ${ }^{18}$ F-FDG brain PET/CT were identified through our radiologic information system. To minimize the potentially confounding effects of concomitant central nervous system disease, patient charts were reviewed to exclude patients with primary or secondary brain neoplasms, epilepsy, collagen vascular disease, neuropsychiatric symptoms such as depression and psychosis, developmental disabilities such as trisomy 21 , and previous brain surgery as well as patients undergoing chemotherapy or taking medications known to interfere with brain metabolism. Most of the identified patients were excluded. The reasons were epilepsy in about 350 patients, brain tumors in about 80 , collagen vascular disease in about 50, encephalitis in about 40, psychiatric disorders in about 10 , thrombotic disease in about 30 , and central nervous system symptoms of uncertain etiology or use of medication in the rest.

After these patients had been excluded, 88 remained: 43 boys and 45 girls, ranging from 6 mo to $18 \mathrm{y}$ old. These were assigned to either a development group $(n=59)$ or a validation group $(n=29)$. We tested for significant differences between the 2 groups using the Pearson $\chi^{2}$ test (for sex) and the Student 2-sample $t$ test (for age).

\section{Imaging}

Before the acquisitions, all patients fasted for a minimum of $4 \mathrm{~h}$. Dextrose-containing intravenous solutions were stopped $2 \mathrm{~h}$ before administration of ${ }^{18} \mathrm{~F}$-FDG. Blood glucose levels were checked before injection to ensure a level no higher than $8 \mathrm{mmol} / \mathrm{L}$.

A $3.5-5 \mathrm{MBq} / \mathrm{kg}$ injection of ${ }^{18} \mathrm{~F}-\mathrm{FDG}$ (minimum, $37 \mathrm{MBq}$ ) was given, and studies were acquired $45 \mathrm{~min}$ afterward for the brain and 90 min afterward for the whole body or head and neck. PET/CT was performed using a Philips Gemini 16 time-of-flight PET/CT scanner. The acquisition duration was $10 \mathrm{~min} /$ field of view for the dedicated brain studies, $3 \mathrm{~min} /$ field of view for the head-and-neck studies, and $2.5 \mathrm{~min} /$ field of view for the whole-body studies. The CT parameters for attenuation correction were $120 \mathrm{kVp}$, a pitch of 0.813 , a rotation time of $0.5 \mathrm{~s}$, a slice thickness of $5 \mathrm{~mm}$, and collimation of $16 \times$ $1.5 \mathrm{~mm}$. The CT tube current was adjusted according to body weight (<45 kg, $22 \mathrm{~mA} ; 46-67 \mathrm{~kg}, 33 \mathrm{~mA} ; 68-90 \mathrm{~kg}, 44 \mathrm{~mA} ;>91 \mathrm{~kg}$, $66 \mathrm{~mA})$. For patients undergoing dedicated brain studies, a current of $45 \mathrm{~mA}$ was used. PET images were acquired in 3-dimensional mode and were reconstructed using a row-action maximum-likelihood algorithm (3 iterations, 33 subsets).

No patients received sedation. Patients undergoing dedicated brain studies were instructed to rest while minimizing auditory and visual stimulation. Other patients were isolated in a dedicated room during the uptake period in the presence of their family and a television.

Prospective identification of head-and-neck or whole-body scans permitting extraction of brain data in patients without central nervous system disease began in October 2015.

\section{Data Analysis}

Brain PET images were analyzed using NeuroQ (Syntermed), a commercially available software package that performs automated quantitative analysis of activity in standardized volumes of interest in the brain. After upper and lower limits were defined for the brain, scalp activity was removed using the threshold set by the program, corresponding to $20 \%$ of the image counts. Slices in 3 planes (axial, coronal, and sagittal) were realigned using rigid registration. Ten iterations were used to produce elastic transformation of the patient brain data to the reference brain template. Regional relative brain metabolism was automatically quantified in 47 standardized cortical and subcortical volumes of interest, including 22 paired regions as shown in Figure 1. Both WBN and PN data were examined. Data loss prevented analysis of one patient with PN.

For each of these regions, the significance of sex was examined using a 2-sample unpaired $t$ test in GraphPad software (https://www. graphpad.com/quickcalcs/ttest1.cfm).

Multiple linear and nonlinear mathematic models were then developed for each region to determine the variation in regional relative brain metabolism with age using CurveExpertProfessional, version 2.6 (Hyams Development). The models considered were linear, quadratic, cubic, logarithmic, power law $\left(y=a x^{b}\right)$, modified power law $\left(y=a b^{x}\right)$, and exponential. The optimal model for each brain region was determined by use of the Akaike information criterion. For each of the 47 brain regions, the optimal model was then analyzed in SPSS24 (IBM Corp.) to obtain the mean predicted value and the $95 \%$ prediction interval as a function of age, using both the WBN and the PN data. A Microsoft Excel program was

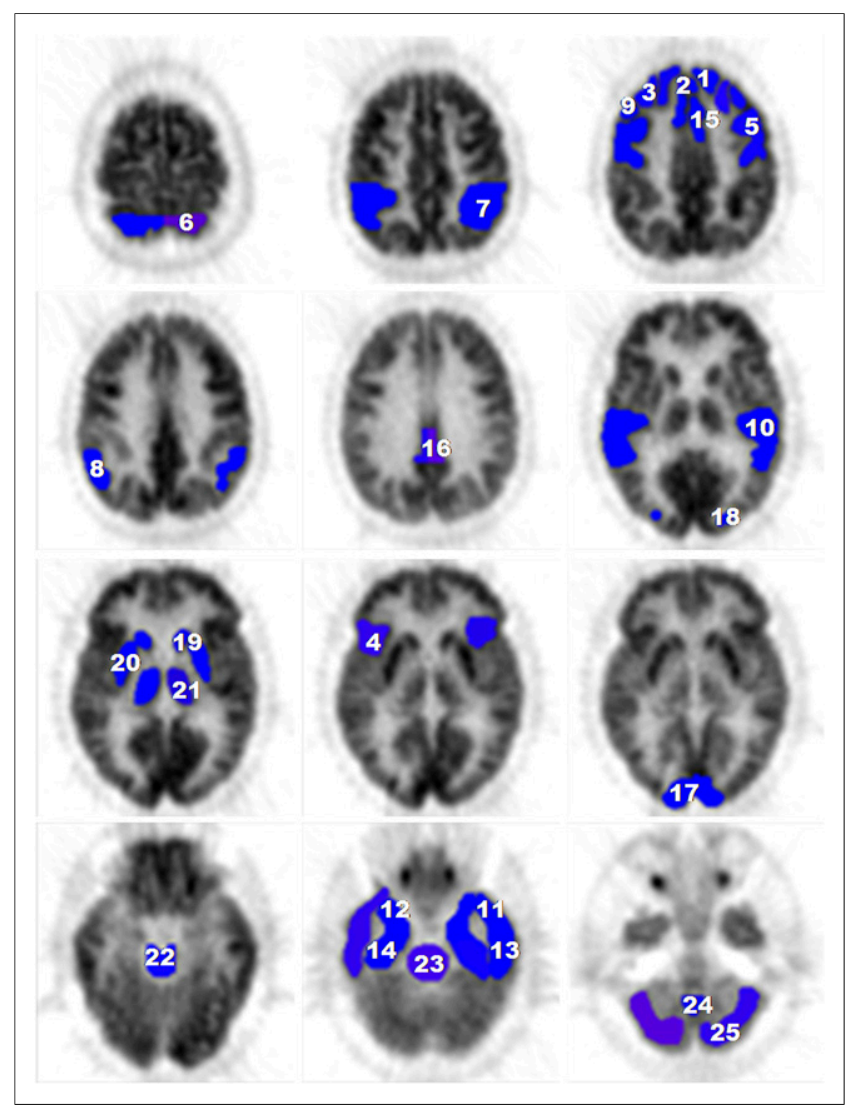

FIGURE 1. Overview of brain segmentation in 47 regions (22 paired) using NeuroQ. 1 = superior frontal (GFs); 2 = medial frontal (GFd); $3=$ mid frontal (GFm); 4 = inferior frontal (GFi); 5 = sensorimotor (SM); $6=$ superior parietal ( $\mathrm{SPL}) ; 7=$ inferior parietal $(\mathrm{iPL}) ; 8=$ parietotemporal (PTC); 9 = Broca; 10 = superior lateral temporal (sLT); $11=$ inferior lateral anterior temporal (iLAT); 12 = anterior medial temporal (MAT); $13=$ inferior lateral posterior temporal (iLPT); $14=$ posterior medial temporal (MPT); 15 = anterior cingulate (GCa); 16 = posterior cingulate (PCC); 17 = primary visual cortex (PVC); 18 = associative visual cortex (AVC); 19 = caudate nucleus (CN); $20=$ lentiform nucleus (LN); $21=$ thalamus (Th); 22 =midbrain (MB); 23 = pons $(\mathrm{P}) ; 24=$ vermis $(\mathrm{V}) ; 25=$ cerebellum (Cbm). (Image Courtesy of Dr. Daniel H. Silverman.) 
TABLE 1

Optimal Models for All Regions Considered in Our Analysis for Both WBN and PN Data

\begin{tabular}{|c|c|c|c|c|c|}
\hline \multicolumn{3}{|c|}{ Left side } & \multicolumn{3}{|c|}{ Right side } \\
\hline Region & WBN model & PN model & Region & WBN model & PN model \\
\hline Frontal lobe & & & Frontal lobe & & \\
\hline GFs & Quadratic & Quadratic & GFs & Quadratic & Quadratic \\
\hline GFd & Power law & Quadratic & GFd & Cubic & Quadratic \\
\hline GFm & Quadratic & Quadratic & GFm & Quadratic & Quadratic \\
\hline Gfi & Logarithmic & Quadratic & GFi & Logarithmic & Quadratic \\
\hline SM & Power law & Quadratic & SM & Power law & Quadratic \\
\hline Parietal lobe & & & Parietal lobe & & \\
\hline $\mathrm{sPL}$ & Logarithmic & Linear & SPL & Linear & Linear \\
\hline $\mathrm{iPL}$ & Linear & Quadratic & $\mathrm{iPL}$ & Linear & Quadratic \\
\hline PTC & Quadratic & Quadratic & PTC & Quadratic & Quadratic \\
\hline Broca & Quadratic & Quadratic & Broca & Logarithmic & Quadratic \\
\hline Temporal lobe & & & Temporal lobe & & \\
\hline sLT & Logarithmic & Quadratic & sLT & Logarithmic & Quadratic \\
\hline iLAT & Logarithmic & Logarithmic & iLAT & Logarithmic & Logarithmic \\
\hline MAT & Quadratic & Logarithmic & MAT & Quadratic & Linear \\
\hline iLPT & Cubic & Linear & iLPT & Cubic & Quadratic \\
\hline MPT & Quadratic & Linear & MPT & Quadratic & Linear \\
\hline Cingulate & & & Cingulate & & \\
\hline GCa & Linear & Logarithmic & GCa & Power law & Quadratic \\
\hline PCC girls & Linear & Quadratic* & PCC girls & Logarithmic & Quadratic* \\
\hline PCC boys & Linear & & PCC boys & Linear & \\
\hline Occipital lobe & & & Occipital lobe & & \\
\hline PVC & Power law & Power law & PVC & Modified power law & Linear \\
\hline AVC & Logarithmic & Linear & AVC & Linear & Linear \\
\hline Basal ganglia & & & Basal ganglia & & \\
\hline $\mathrm{CN}$ & Logarithmic & Logarithmic & $\mathrm{CN}$ & Modified power law & Quadratic \\
\hline LN & Power law & Quadratic & $\mathrm{LN}$ & Power law & Quadratic \\
\hline Th & Cubic & Cubic & Th & Cubic & Cubic \\
\hline Posterior fossa & & & Posterior fossa & & \\
\hline Cbm & Logarithmic & Power law & $\mathrm{Cbm}$ & Linear & Power law \\
\hline Vermis & Cubic & Power law & & & \\
\hline $\mathrm{MB}$ & Quadratic & Logarithmic & Pons & Quadratic & \\
\hline
\end{tabular}

*Same model applies for girls and boys.

Key to region abbreviations is in legend of Figure 1.

developed allowing direct transfer of NeuroQ data, sex and age, and the comparison of the mathematic model predictions to the validation group. The percentages of regions within and beyond the $95 \%$ prediction interval were calculated. The error in prediction for each region and patient was calculated as the absolute value of [(predicted value - validation data)/ predicted value $\times 100$ ] using the appropriate $\mathrm{WBN}$ and PN validation data, averaged over all validation patients for each region and defined as the regional error. The mean error in prediction was calculated as the mean of all regional errors.

Univariate comparisons between the $\mathrm{WBN}$ and $\mathrm{PN}$ validation data for the number of regions within, below, or above the $95 \%$ prediction interval and the mean errors in prediction were performed using a 2 sample paired $t$ test.

\section{RESULTS}

\section{Demographics}

The development group included 30 boys (mean age \pm SD, $9.15 \pm$ $5.6 \mathrm{y})$ and 29 girls $(9.83 \pm 4.8 \mathrm{y})$, and the validation group included 13 boys $(8.77 \pm 5.4 \mathrm{y})$ and 16 girls $(11 \pm 5.4 \mathrm{y})$. There was no significant difference in age between male and female subjects in the development group $(P=0.62)$ or the validation group $(P=0.28)$, and there was no significant difference in age $(P=0.67)$ or sex $(P=0.59)$ between the development and validation groups.

In 13 patients, the brain PET slices were obtained from a dedicated brain study; in 43, from a head-and-neck acquisition; and in 32, from a whole-body acquisition. Dedicated head-and-neck acquisitions were 


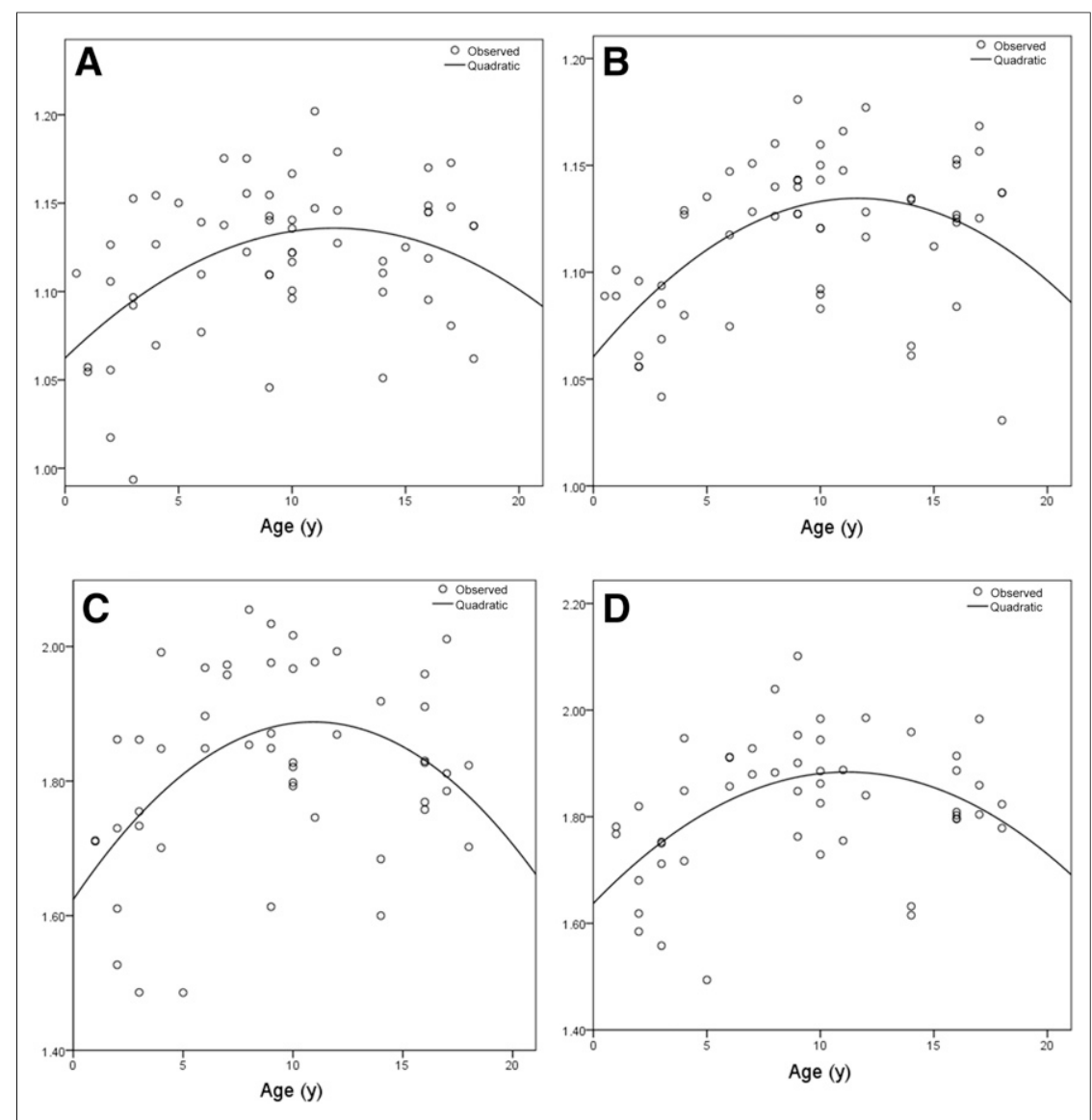

FIGURE 2. Optimal quadratic models of regional relative brain metabolism for middle frontal gyri: WBN data for right (A) and left (B) sides, and PN data for right (C) and left (D) sides. using $\mathrm{PN}$, with a maximal ratio to whole brain or pons activity noted at around 10-12 y of age. Selected regions are demonstrated in Figure 2. In the nonquadratic models, activity increased with age with the exception of the sensorimotor cortex, which demonstrated decreasing values in the WBN model.

In the parietal lobes, for the PN data 6 of the 8 models were quadratic, with these regions peaking around $10 \mathrm{y}$ of age. For the WBN data, only 3 regions followed a quadratic model, with a maximum reached at $9 \mathrm{y}$ of age. For the remaining regions, the ratio of activity decreased linearly as a function of age in 3 regions for WBN and in 2 for PN. Selected regions are shown in Figure 3.

In the temporal lobes, functional dependence on age varied significantly among the 10 regions considered. In the medial temporal regions, activity decreased with age, with 4 regions being fitted with a quadratic model and a minimum being reached between the ages of 10 and $14 \mathrm{y}$. In most of the models for the lateral temporal regions, increasing activity was found but was generally modest. In the 3 regions fitted with a quadratic model, the maximum was reached around the age of $10 \mathrm{y}$.

Results in the occipital lobe showed activity that decreased with age for both the WBN and the PN data; however, the decrease was more marked for WBN than

performed more often in older patients, whereas whole-body acquisitions were more common in younger patients.

In 13 patients, the PET study was performed to evaluate neoplasms; in 34, to evaluate lymphadenopathy (including lymphoma); in 20, to evaluate fever and infection; and in the remainder, to evaluate miscellaneous indications (Supplemental Tables 1 and 2; supplemental materials are available at http://jnm.snmjournals.org).

\section{Effect of Sex}

For the WBN data, the effect of sex on local brain metabolism was not statistically significant $(P=0.13-0.95)$ in any region assessed, with the exception of the posterior cingulate gyri $(P=0.028$ and 0.032 for the right and left, respectively). For the PN data, the effect of sex was not significant in any region $(P=0.16-0.49)$.

\section{Evolution of Local Brain Metabolism with Age}

In terms of age, the optimal model for the WBN data was linear in 9 regions, quadratic in 13 , cubic in 6 , logarithmic in 12 , power law in 7, and modified power law in 2 (Table 1; Supplemental Table 3). For the PN model, the optimal dependence on age was linear in 9 regions, quadratic in 25 , cubic in 2 , logarithmic in 6 , and power law in 4 (Table 1; Supplemental Table 4). The same model applied for the right and left sides in 14 of 22 paired regions for the WBN models and 17 for the PN models. No region demonstrated constant activity with age.

In the frontal lobes, 4 regions were best fitted with a quadratic model using WBN, and all were best fitted with a quadratic model for PN. In the 4 nonlinear models fitted in the occipital lobe, the sharpest decrease occurred between 0 and 2 y of age.

The cerebellar hemispheres showed a continuous increase with age. Using WBN, the optimal model was a power law for the left cerebellum and linear for the right. Using PN, the model was a power law for both cerebellar hemispheres, and the parameters were similar. The midbrain was represented by a quadratic model using WBN, with a minimum reached at $10 \mathrm{y}$ of age, and by a positive logarithmic equation using PN.

Activity in the basal ganglia and thalamus showed the most variation in models and in trends. The lentiform nuclei were decreasing slowly with age, but by a quadratic model using PN, with a maximum reached around age $10 \mathrm{y}$ and similar parameters. The left caudate nucleus was modeled with a logarithmic equation using WBN and PN data, with activity slightly increasing with age. However, for the right caudate nucleus, the model was a modified power law using WBN and quadratic using PN, with a slight decrease in activity with age. Thalamic activity was represented by the cubic model for both WBN and PN. The variation in thalamic activity with age is shown in Figure 4.

\section{Comparison to the Validation Group}

When comparing the model predictions with our validation group, we found that the activity in $1,286(94.3 \%)$ of the 1,363 regions in our validation group fell within the $95 \%$ prediction interval, with 50 regions below and 26 above the predicted range represented by a power law model using WBN, with activity 


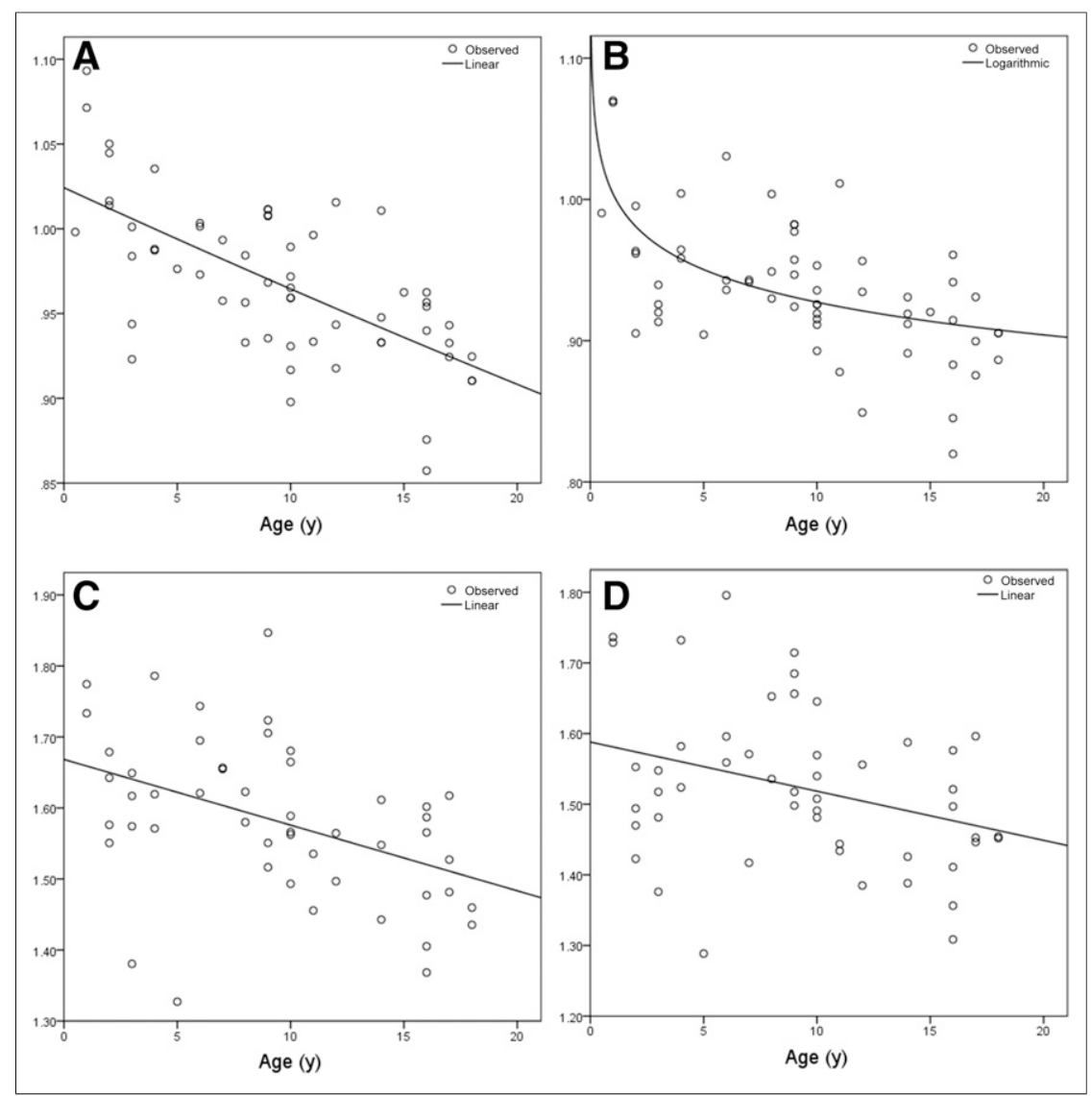

FIGURE 3. Optimal models of regional relative brain metabolism for superior parietal gyri: WBN linear model for right side (A) and WBN logarithmic model for left side (B); PN linear model for right side (C) and PN linear model for left side (D).

using WBN data. For the PN validation data, the activity in 1,210 of the 1,288 regions (94\%) fell within the $95 \%$ prediction interval, with 59 regions below and 19 above the predicted interval. In the 4 patients aged $2 \mathrm{y}$ or less, there were 26 regions outside the $95 \%$ prediction interval for the WBN data and 48 for the PN data. There was no significant difference between the WBN model and the PN model in the number of regions below $(P=0.8)$ or above $(P=0.47)$ the $95 \%$ predicted interval or in the total $(P=0.97)$.

When comparing the models with our validation group data, we found that regional error varied from $1.79 \%$ to $5.48 \%$ for $\mathrm{WBN}$, as shown in Figure 5A, and from $2.35 \%$ to $8.06 \%$ for PN, as shown in Figure 5B. For all brain regions in the validation group, the mean percentage error in prediction was $3 \% \pm 0.96 \%$ for $\mathrm{WBN}$ and $4.72 \% \pm 1.25 \%$ for $\mathrm{PN}(P<0.0001)$.

\section{DISCUSSION}

Our results demonstrate that it is feasible to accurately model the evolution of normal pediatric regional relative brain metabolism as a function of age and sex. Our models showed excellent agreement with our validation data. We have shown that, when normalized either to whole-brain activity or to a specific region (i.e., pons), regional relative brain metabolism in all cortical and subcortical regions evolves with age. Furthermore, we have shown that sex differences, although present, have a very limited effect on the evolution of regional relative brain metabolism. Qualitatively, our results were comparable to those seen in other studies, showing a posterior-to-anterior shift in regional relative brain metabolism with age, with the degree of activity in the frontal lobes being relatively attenuated during the initial years of life, gradually increasing, and peaking in late prepubescence $(4,9,10)$.

A few studies have examined the evolution of regional relative brain metabolism in childhood. Most of those using dedicated brain PET were performed on patients with epilepsy or suspected neurologic anomalies $(1,5,10,15)$. Others restricted their attention to patients over a limited age range $(2,7)$. Only a few studies, including the current one, examined relatively large numbers of neurologically normal patients $(3,6,9)$. Nonetheless, all these studies are broadly concordant $(8,16)$ and demonstrate a stereotypical pattern of evolution of brain metabolism. Brain metabolism in the neonate is concentrated mainly in the regions responsible for basic functions-primary motor and sensory areas, basal ganglia, brain stem, and vermis $(1,2)$. Maturation of regions related to spatial orientation, language, and attention follows. Prefrontal cortices are among the last regions to mature, including those responsible for attention, coordination, executive function, and emotional control. In late adolescence, "synaptic pruning" occurs $(8,16)$. This may explain the quadratic fitting of many of our regions, with peak age around 10-12 y, similar to other studies $(1,5,9,10)$.

For some brain regions, the functional form of the models differed between the left and right hemispheres, likely because of small differences in the goodness of fit of the models rather than a significant difference in the evolution of the metabolism in contralateral regions. For example, in some regions there were minimal differences between the linear, power law, modified power law, and logarithmic models (Supplemental Tables 3 and 4).

The use of regional relative brain metabolism obviates arterial blood sampling but introduces an ambiguity related to the choice of normalization. Various authors have attempted different approaches - with normalization to the cerebellum (9), both maximum cerebral cortical uptake and basal ganglia (3), or the average count per voxel (6) - and it is not clear which approach is most appropriate. Among the reference structures available with NeuroQ, we chose to normalize to whole-brain activity, as other authors have done, as well as to the pons. The latter was chosen on the basis of its being a midline structure, hence avoiding any potential issues of laterality based on handedness or diaschisis. When comparing the validation data to the mathematic models, we found that the number of regions falling outside the $95 \%$ prediction interval was similar between the WBN and PN models. The error in prediction was significantly lower when models were derived using WBN. However, comparisons to PN data may be useful in patients with diffusely decreased cortical activity, such as in patients who are sedated or have anatomic defects. Although the resultant quantitative models differed, the evolution of metabolism in many brain regions was qualitatively similar for both the WBN and PN data. As 


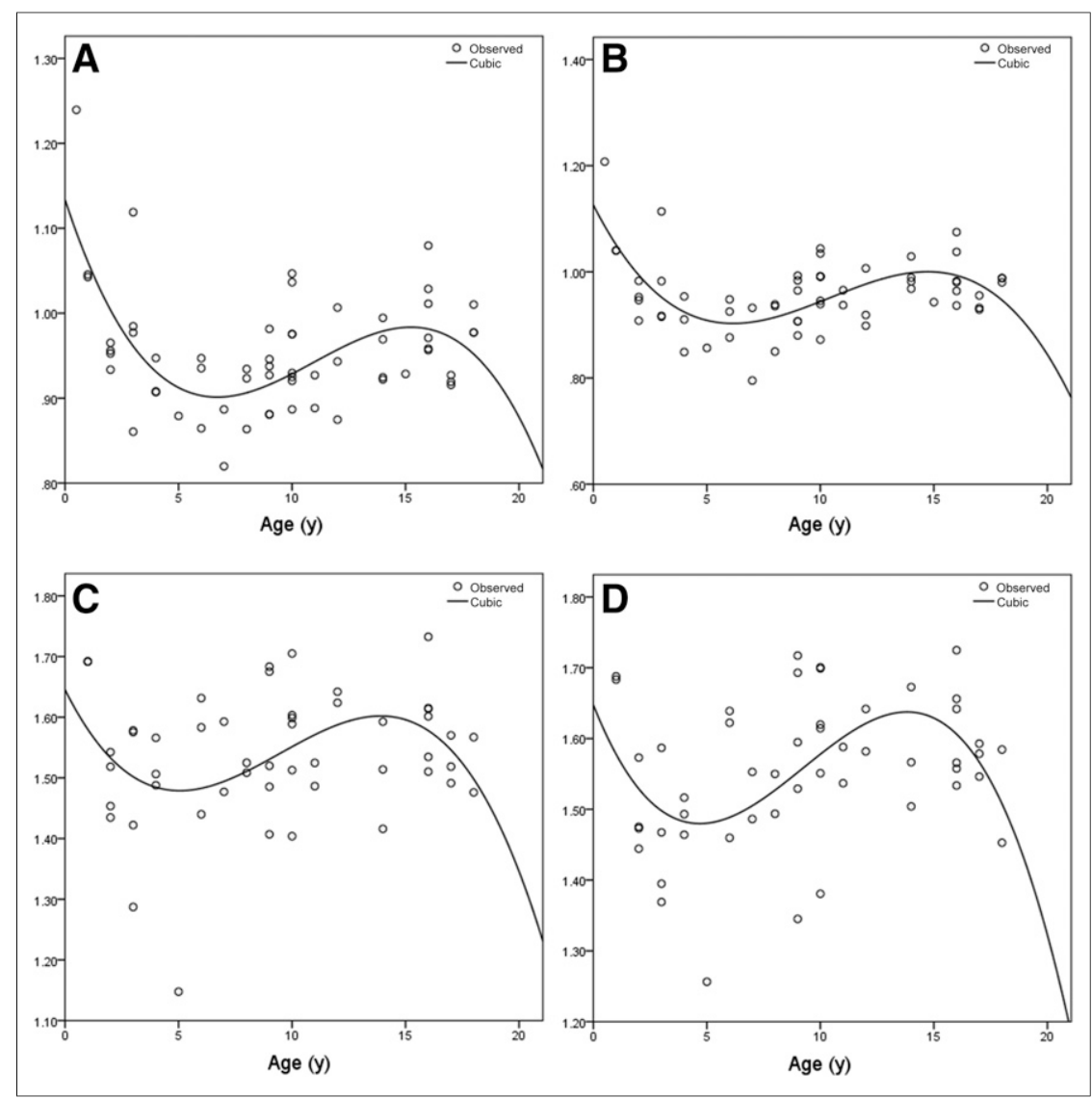

FIGURE 4. Optimal cubic models of regional relative brain metabolism for thalamus: WBN data for right $(A)$ and left $(B)$ sides, and $P N$ data for right $(C)$ and left $(D)$ sides.

such, further study will be required to determine the optimal approach to normalization of brain metabolic activity.

This study was subject to several limitations. Fitting pediatric brains to a standardized adult template may have introduced systematic errors due to misregistration and elastic transformation of the small children's brains. For WBN about $33 \%$ of the regions outside the $95 \%$ prediction interval, and for PN about $60 \%$, were found in patients 2 y or younger. In particular, it is known that the goodness of fit of the spatial normalization of a pediatric brain to an adult template is age-dependent and is particularly relevant in very young patients, especially those under 6 y old (17-19). This fit could have been improved through the use of a custom normalization template (20). However, the current version of our software prevented us from doing so or from directly creating our own reference database. In addition, certain clinical information, such as handedness, could have been considered and included in the models.

Another significant limitation of our study is the small number of dedicated brain studies, as well as the use of patients with systemic disease as surrogates for "healthy" patients. None of the children underwent a formal neuropsychological evaluation, but their medical records were reviewed carefully to identify any anomaly that might affect brain metabolism. Both of these limitations stem from restrictions on scanning healthy pediatric subjects. The larger voxel size $\left(4 \mathrm{~mm}^{3}\right)$ of whole-body and head-and-neck acquisitions, when compared with the smaller voxel size $\left(2 \mathrm{~mm}^{3}\right)$ of dedicated brain studies, may also be a limitation (21). Nonetheless, it is unclear to what extent our results would have been modified if most of our patients had received dedicated brain acquisitions.

There are numerous contexts in which these models can be clinically relevant. In particular, these models provide an age and sex reference standard that can then be used to aid in the interpretation of individual pediatric ${ }^{18} \mathrm{~F}$-FDG PET brain studies for patients with central nervous system disease. For example, these models can be directly applicable to the interpretation of epilepsy studies $(12,22)$, neurooncology cases, or patients with infectious or inflammatory brain diseases such as encephalitis, for either diagnosis or follow-up $(23,24,25)$. Our method offers an alternative to the more time-consuming and technically challenging use of statistical parametric mapping $(4,5,7,22,23)$. However, further work is required to better determine the optimal use of these models.

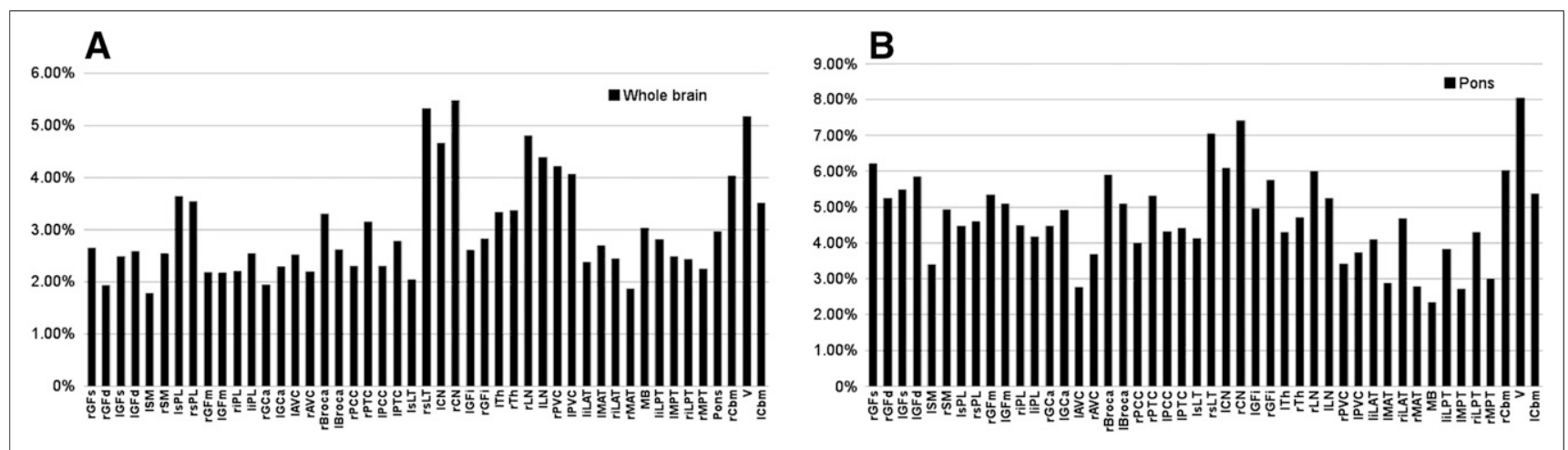

FIGURE 5. Absolute error in regional relative brain metabolism predicted by our models compared with validation group for WBN data (A) and PN data (B). Key to region abbreviations is in legend of Figure 1; "r" and "I" before abbreviation indicate right and left, respectively. 


\section{CONCLUSION}

To our knowledge, this was the first study to use a large number of neurologically intact patients to develop and validate models of regional relative brain metabolism in children, and these models were accurate over the entire pediatric age range (0-18 y). Furthermore, we have examined the effect of using both WBN and PN data and found that WBN data led to more accurate models. In addition, we considered broader classes of mathematic models than those presented in similar studies.

\section{DISCLOSURE}

This project was supported by the 2016 10th Faculty Competition of the Radiology, Radio-Oncology, and Nuclear Medicine Department, Faculty of Medicine, University of Montreal, Montreal, Quebec, Canada. No other potential conflict of interest relevant to this article was reported.

\section{ACKNOWLEDGMENTS}

We thank Dr. Daniel H. Silverman (NeuroQ) and Daniel G. Hyams (CurveExpert).

\section{REFERENCES}

1. Chugani HT, Phelps ME, Mazziotta JC. Positron emission tomography study of human brain functional development. Ann Neurol. 1987;22:487-497.

2. Kinnala A, Suhonen-Polvi H, Aärimaa T, et al. Cerebral metabolic rate for glucose during the first six months of life: an FDG positron emission tomography study. Arch Dis Child Fetal Neonatal Ed. 1996;74:F153-F157.

3. London K, Howman-Giles R. Normal cerebral FDG uptake during childhood. Eur J Nucl Med Mol Imaging. 2014;41:723-735.

4. London K, Howman-Giles R. Voxel-based analysis of normal cerebral $\left({ }^{18} \mathrm{~F}\right) \mathrm{FDG}$ uptake during childhood using statistical parametric mapping. Neuroimage. 2015;106:264-271

5. Van Bogaert P, Wikler D, Damhaut P, Szliwowski HB, Goldman S. Regional changes in glucose metabolism during brain development from the age of 6 years. Neuroimage. 1998;8:62-68.

6. Hua C, Merchant TE, Li X, Li Y, Shulkin BL. Establishing age-associated normative ranges of the cerebral ${ }^{18} \mathrm{~F}-\mathrm{FDG}$ uptake ratio in children. $\mathrm{J} \mathrm{Nucl} \mathrm{Med}$. 2015;56:575-579.

7. Kim I-J, Kim S-J, Kim Y-K. Age- and sex-associated changes in cerebral glucose metabolism in normal healthy subjects: statistical parametric mapping analysis of F-18 fluorodeoxyglucose brain positron emission tomography. Acta Radiol. 2009;50:1169-1174.

8. Gogtay N, Giedd JN, Lusk L, et al. Dynamic mapping of human cortical development during childhood through early adulthood. Proc Natl Acad Sci USA. 2004;101:8174-8179.
9. Shan ZY, Leiker AJ, Onar-Thomas A, et al. Cerebral glucose metabolism on positron emission tomography of children. Hum Brain Mapp. 2014;35:22972309.

10. Bentourkia M, Michel C, Ferriere G, et al. Evolution of brain glucose metabolism with age in epileptic infants, children and adolescents. Brain Dev. 1998;20: 524-529.

11. Mosconi L, Tsui WH, Pupi A, et al. ${ }^{18}$ F-FDG PET database of longitudinally confirmed healthy elderly individuals improves detection of mild cognitive impairment and Alzheimer's disease. J Nucl Med. 2007;48:1129-1134.

12. Drzezga A, Arnold S, Minoshima S, et al. ${ }^{18}$ F-FDG PET studies in patients with extratemporal and temporal epilepsy: evaluation of an observer-independent analysis. J Nucl Med. 1999;40:737-746.

13. Patterson JC, Lilien DL, Takalkar A, Pinkston JB. Early detection of brain pathology suggestive of early AD using objective evaluation of FDG-PET scans. Int J Alzheimers Dis. 2010;2011:946590.

14. Perani D, Della Rosa PA, Cerami C, et al. Validation of an optimized SPM procedure for FDG-PET in dementia diagnosis in a clinical setting. Neuroimage Clin. 2014;6:445-454.

15. Martineau P, Lambert R, Décarie J, Turpin S. Investigating the utility of FDGPET/CT for pediatric encephalitis. [abstract] Eur J Nucl Med Mol Imaging. 2016;43(suppl):S63.

16. Marsh R, Gerber AJ, Peterson BS. Neuroimaging studies of normal brain development and their relevance for understanding childhood neuropsychiatric disorders. J Am Acad Child Adolesc Psychiatry. 2008;47:1233-1251.

17. Muzik O, Chugani DC, Juhasz C, Chengang S, Chugani HT. Statistical parametric mapping: assessment of application in children. Neuroimage. 2000;12:538549.

18. Lenroot RK, Gogtay N, Greenstein DK, et al. Sexual dimorphism of brain developmental trajectories during childhood and adolescence. Neuroimage. 2007; 36:1065-1073.

19. Mandal PK, Mahajan R, Dinov ID. Structural brain atlases: design, rationale, and applications in normal and pathological cohorts. J Alzheimers Dis. 2012;31 (suppl):S169-S188.

20. Fonov V, Evans AC, Botteron K, Almli CR, McKinstry RC, Collins DL. Unbiased average age-appropriate atlases for pediatric studies. Neuroimage. 2011; 54:313-327.

21. Kolthammer JA, Su KH, Grover A, Narayanan M, Jordan DW, Muzic RF. Performance evaluation of the Ingenuity TF PET/CT scanner with a focus on high count-rate conditions. Phys Med Biol. 2014;59:3843-3859.

22. Verger A, Yagdigul Y, Van Der Gutch A, et al. Temporal epilepsy lesions may be detected by the voxel-based quantitative analysis of brain FDG-PET images using original block matching normalization software. Ann Nucl Med. 2016; 30:272-278.

23. Archambaud F, Bouilleret V, Hertz-Pannier L, et al. Optimizing statistical parametric mapping analysis of ${ }^{18} \mathrm{~F}-\mathrm{FDG}$ PET in children. EJNMMI Res. $2013 ; 3: 2$

24. Solnes LB, Jones KM, Rowe SP, et al. Diagnostic value of ${ }^{18}$ F-FDG PET/CT versus MRI in the setting of antibody-specific autoimmune encephalitis. J Nucl Med. 2017;58:1307-1313.

25. Morbelli S, Arbizu J, Booij J, et al. The need of standardization and of large clinical studies in an emerging indication of ${ }^{18} \mathrm{~F}$-FDG PET: the autoimmune encephalitis. Eur J Nucl Med Mol Imaging. 2017;44:353-357. 